\title{
Large hoisting machinery local damage acoustic emission monitoring of optical information acquisition research
}

\author{
Shuai WAN, Qing LI, ${ }^{a}$ and Xian-Jin WANG \\ College of Mechanical and Electrical Engineering, China Jiliang University, Hang Zhou, Zhe Jiang, China \\ a Corresponding author:Qing Li E-mail:Iq13306532957@163.com
}

\begin{abstract}
AE technology,an advanced fault diagnosis technique,is impacted by environmental noise during monitoring equipment. The occurrence of interfere noise, the fashion of interfere and the effect to the system in the $\mathrm{AE}$ automatic online monitoring system is analyzed. At present, most of the acoustic emission signal transmission by cable, this way of transmission has a limited transmission distance, shortcomings and so on signal easily disturbed. Is proposed in this paper based on the optical fiber transmission technology, designed and developed a information collection system based on optical fiber acoustic emission monitoring. This way has the advantages of long distance transmission, strong anti-jamming capability.
\end{abstract}

\section{Guideline}

At present, the safety guarantee of the hoisting machinery in the operation process mainly depends on the checking and routine maintenance. And the fixed time interval of the inspection, and the conventional non-destructive testing technology only detecting some geometric and physical quantities of the lifting machine, and can not be detected under the condition of the lifting machine. Lack of hoisting machinery operating state for the related factors of safety monitoring, not of hoisting machinery health to achieve the real-time and comprehensive grasp, also do not track crane damage propagation and damage alarming. Lead to heavy machinery often pass the conventional test, but in the normal operating conditions are still likely to occur structural failure, crack growth and other damage caused by major accidents[1].Acoustic emission technique is the main method to detect large equipment active defects, such as crack propagation, but due to the complexity of the work site of the large equipment, harsh environment, traditional acoustic emission technology was influenced by the physical properties of cable transmission inherent, with transmission distance is limited, vulnerable to interference and other shortcomings, for traditional acoustic emission monitoring difficult.Therefore, this paper proposes to replace the traditional cable with optical fiber transmission signal, not only can achieve long-distance transmission and greatly reduce the external interference and signal attenuation.

\section{Principle of acoustic emission detec -tion}

Acoustic emission (AE), also known as stress wave emission is materials or components by external force deformation, fracture or internal stress exceeds the yield limit and enter the irreversible plastic deformation stage, the transient elastic wave in the form of the release of the strain energy of the phenomenon. Since acoustic emission testing, in the key part of the equipment, generally choose design stress value is larger or prone to corrosion, cracks or actual use of defects (such as cracks) parts of the arrangement of sensors had occurred in the process. During the normal operation of the hoisting machine, the source of corrosion and crack in the material will produce acoustic emission (stress wave) signal, which contains information of serious structural defects [2].Acoustic emission detection principle as shown in Fig.1, from the acoustic emission source to produce the wave reaching the material surface, causing the surface displacement, the sensor will mechanical vibration of materials conversion into electrical signals and are then amplified, processing and recording. Based on the observed acoustic emission signals, the mechanism of acoustic emission is analyzed and deduced. 


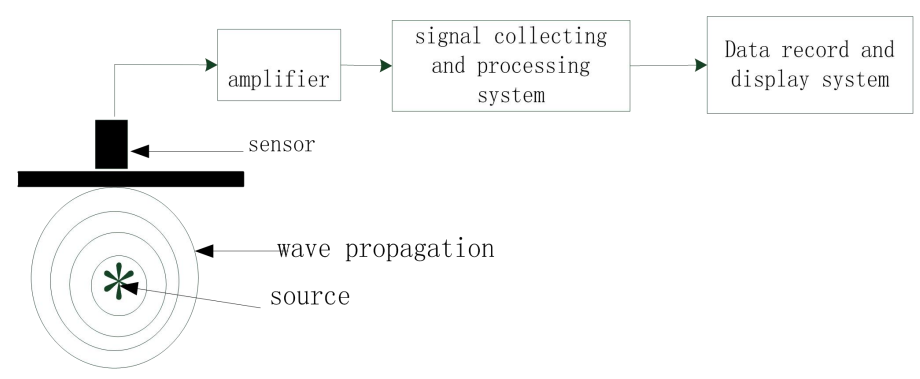

Fig .1 Principle of Acoustic Emission Detection

The frequency range of acoustic emission wave can be from a few $\mathrm{Hz}$ to several thousand $\mathrm{MHz}$, which is related to the properties of the material, the microstructure of the sound source and the micro process of the release energy. The larger the size of the acoustic emission source, the faster the frequency, the wider the frequency range. Different acoustic emission mechanism of the spectrum is different, corresponding to the amplitude of various frequency components are also different [2].

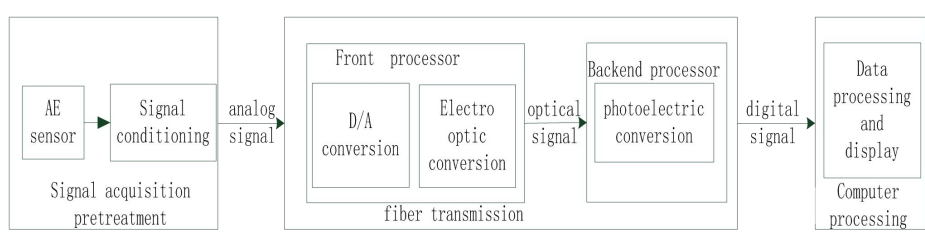

Fig.2 Acoustic Emission Optical Fiber Information Acquisition of System

\section{Acoustic emission fiber information acquisition of system}

As Fig.2, the acoustic emission optical fiber information acquisition of system is composed of four parts, which are acoustic emission sensor, data acquisition module, optical fiber transmission module and PC software system.

\subsection{Signal acquisition pretreatment}

Signal acquisition and preprocessing mainly by acoustic emission sensor and signal conditioning is composed of two parts, the acoustic emission sensors to collect acoustic emission signal, which is faint, require a signal conditioning of the conditioning.Because the signal received by the acoustic emission sensor is relatively weak, the first to be amplified, and then the analog signal conditioning for the A/D chip can receive the standard signal. Finally, the real-time acoustic emission is completed by the FPGA control, the feature extraction and acoustic emission waveform acquisition. As the output signal of the sensor is very weak, it is necessary to reduce the signal to noise ratio in the long distance transmission. Therefore, it is necessary in close proximity to the sensor placement of the front-end amplifier, according to a certain ratio of signal amplification to a level suitable for subsequent processing module processing,[3] then the output amplification analog signal by adjusting the transmission to the signal processing unit (capture card) for processing.Front end amplifier is an analog circuit and the working power supply of the power supply, signal collinear way transportation, working principle as shown in Fig.3.

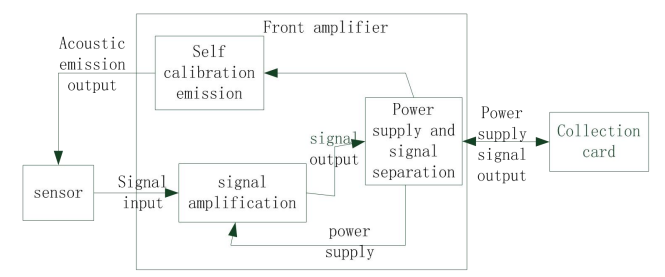

Fig.3 Working Principle Diagram

\subsection{Fiber transmission module}

The optical signal is transmitted by the optical fiber transmission system, so the analog signal transmitted by the acquisition card is converted into a digital signal, and the digital signal is modulated by the digital signal. In the optical fiber transmission system, the change of the electrical signal with the information is added to the optical carrier, which makes the optical carrier change according to the change of the information. This is the modulation of the optical wave [4].Speaking from the essence, the light carrier modulation and radio carrier modulation as, can carry the signal amplitude, intensity, frequency, phase and polarization parameters make light to carry information, with amplitude modulation (AM), intensity modulation, frequency modulation, phase modulation, the modulated offset multiple modulation. But in order to facilitate the demodulation, the intensity modulation mode is adopted in the optical frequency band.The back end of the optical fiber transmission needs to be photoelectric demodulation. The task of the photoelectric demodulation module is to recover the information carried by the optical fiber transmission and optical carrier with the minimum additional noise and distortion. The front end of the photoelectric demodulation module includes a photodiode and a 
preamplifier [5] under reverse bias.A photodiode receives an optical signal coupled to an optical fiber. In actual circuit analysis, the photodiode can be regarded as a current source in parallel with its junction capacitance $C_{d}$, equivalent circuit is shown in Fig.4, where $R_{L}$ is the load resistance.

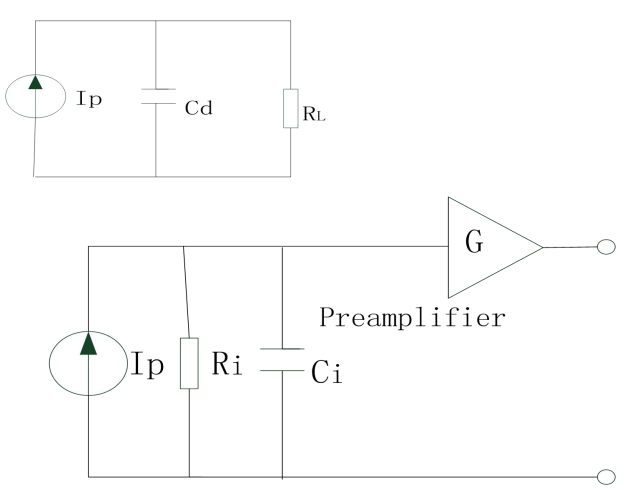

Fig.4 Photo Diode Equivalent Circuit Diagram

The design of the front end of the photoelectric demodulation module is shown in Fig. 5. $\mathrm{Ci}$ for the total input capacitance, which includes the junction capacitance of the photodiode and pre amplifier transistor induced capacitance.

Fig.5 Photoelectric Demodulation Front-End Circuit

In the high impedance preamplifier, due to the input circuit of the total resistance $R_{i}$ is larger, can increase the input voltage of the preamplifier, the larger $R_{i}$ values can also reduce the thermal noise and increased receiver sensitivity, but the drawback is bandwidth delta $f$ is narrow. The bandwidth of this circuit can be expressed as

$$
\triangle f=\left(2 \pi R_{i} C_{i}\right)^{-1}
$$

The total resistance of the input circuit $\mathrm{R}_{\mathrm{i}}$ is connected in parallel with the input resistance $R_{b}$ of the amplifier and the $\mathrm{D}_{\mathrm{C}}$ load resistance $\mathrm{R}_{\mathrm{L}}$ of the photodiode. Equivalent input resistance $R_{i}$ is expressed as

$$
\mathrm{Ri}=\mathrm{Rb} / / \mathrm{RL} \text {. }
$$

The thermal noise introduced by the input circuit is expressed as

$$
\mathrm{E} 2 \mathrm{Rn}=4 \mathrm{kTRi} \triangle \mathrm{f} .
$$

As can be seen, the greater the $\mathrm{R}_{\mathrm{L}}$, the smaller the bandwidth. The equalizer can be used to increase the bandwidth of the high frequency band,[6] and it can be used to reduce the $R_{i}$ to increase the bandwidth when the receiver sensitivity is up to the requirement.

\subsection{Software design and development}

In view of the requirement of acoustic emission optical fiber information acquisition system, the acoustic emission information acquisition software is developed, which is used in visual studio2010 development environment, which provides a large number of controls to facilitate the development of the system.Acoustic emission optical fiber information acquisition system mainly consists of four parts, such as data acquisition module, parameter setting module, feature parameter extraction module, historical data query module and so on. The block diagram of the software system is shown in Fig.6.

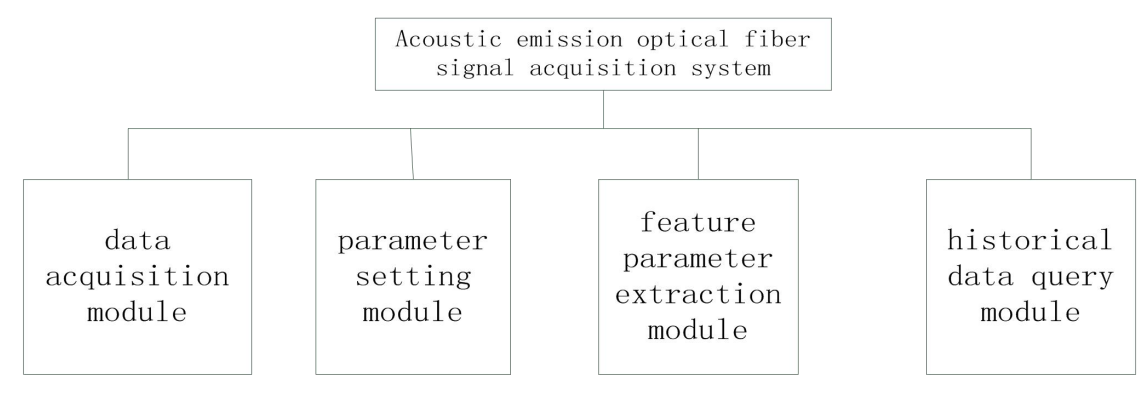

Fig.6 Block Diagram of the Software System

\section{Data acquisition experiment}

In order to validate the reliability of fiber acquisition system emission, in the process of tensile specimen design acoustic emission signal monitoring experiment, using acoustic emission (AE) fiber collection system and the traditional acoustic emission detection system to detect the sample at the same position, through comparative analysis to verify the acoustic emission (AE) optical fiber acquisition system whether to replace the acoustic emission signal transmission cable. 


\subsection{Experimental apparatus and materials}

The test equipment mainly includes: steel test specimens, tensile testing machine, acoustic emission fiber acquisition system, PC software and so on.

The sample is made of steel Q235-B, plate thickness is $4 \mathrm{~mm}$, and the standard specimen of long $340 \mathrm{~mm}$ is shown in Fig.7.

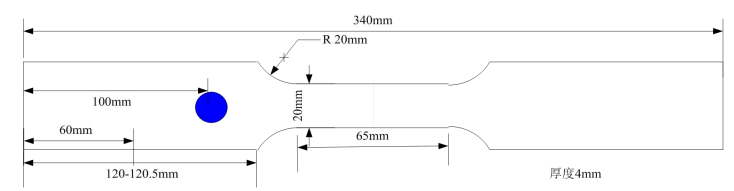

Fig.7 Schematic Diagram of The Sample (Round For The Sensor Installation Location)

\subsection{Experimental procedure}

Before the experiment, first of all, on the test machine electric clamping test pieces and to adjust the clamping distance of the test piece, set aside the location of the sensor installation. Connection monitoring equipment, monitoring system of optical fiber transmission distance of about $500 \mathrm{~m}$, through experimental lead, in the surface of the specimen broken lead core simulation of acoustic emission source, test the sensor amplitude of the two sets of system response and calibration of the sensor sensitivity.

First measurement of the field environmental noise, according to the measured noise value $(40 \mathrm{~dB})$, the threshold value is set to $45 \mathrm{~dB}$, lower than the $45 \mathrm{~dB}$ signal will be treated as noise filter out, the system sampling $10 \mathrm{MSPS}$, length $2 \mathrm{~K}$. To start the tensile testing machine,

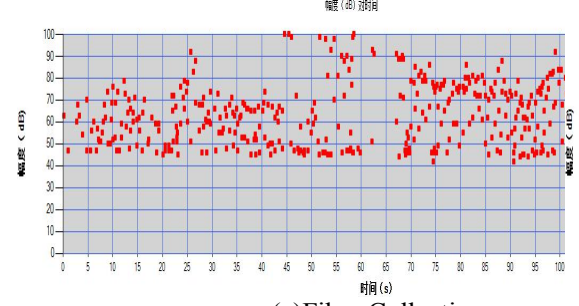

(a)Fiber Collection

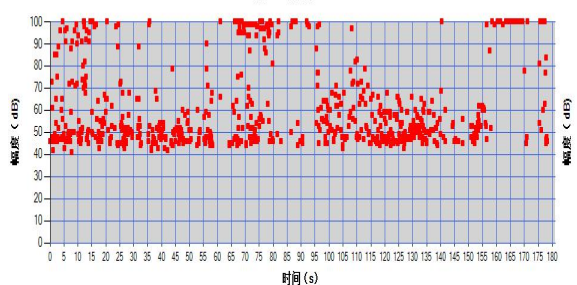

(c)Fiber Collection the tensile testing machine is set up to $5 \mathrm{~mm} / \mathrm{min}$. After the start, the tension machine is loaded $(5 \mathrm{~mm} / \mathrm{min})$ according to the predetermined loading speed, until the specimen is stretched and broken. The acoustic emission monitoring system records the data of the whole process, and the test device is shown in Fig.8.

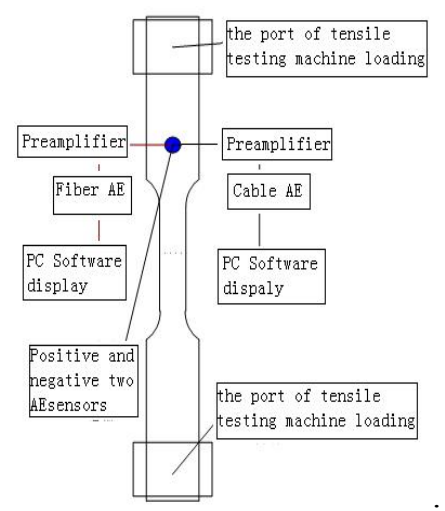

Fig.8 Test Equipment Diagram

\subsection{Data comparison and analysis}

When the tensile specimen after the cessation of monitoring, monitoring system records the tensile process of signal. The amplitude of the 2 samples is shown in Fig.9 of the time.The horizontal axis is time, the vertical axis is amplitude.Amplitude: the maximum amplitude of the event signal waveform, not affected by the threshold. Usually with $\mathrm{dB}$ (sensor output $1 \mathrm{~V}$ to $0 \mathrm{~dB}$ ).

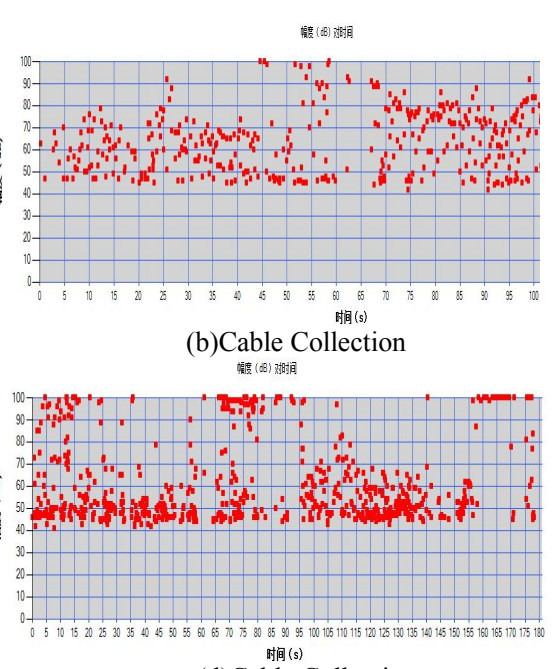

(d)Cable Collection

Fig.9 Amplitude to Time Scatter Plot Comparison Diagram

From the above comparison shows that acoustic emission fiber information acquisition system and traditional cable acquisition results are basically the same, predictably, acoustic emission fiber information acquisition system which can effectively extend the monitoring distance and strong anti-interference ability, can realize the long-distance monitoring of local damage.

\section{Conclusion}

Verified by experiment, the system can adapt to long distance acoustic emission monitoring tasks under the complex environment of long distance transmission of signals. Acoustic emission optical fiber acquisition technology provides a powerful technical means to carry 
out the monitoring of long distance structural defects, and has broad application prospects.

\section{References}

[1]. LiLi,Chen,Xiang-qian Zhao.Meiyun.Acoustic EmissionSignal Characteristics of Active Defects in Crane[J].Nondestructive Testing,2008,30（6）:334 337.

[2]. ShengGongtian,Dai Guang,Liu Shifeng.Development of Acoustic Emission Detection Technology in China[J].Nondestructive Testing,2003,25(6):302-307.

[3]. Liu Juan,Ding Keqing.Research Status and Prospect of Optical Fiber Acoustic Emission Sensor[J].Sensors and Micro Systems, 2010,29(9): 5-7.

[4]. Li Wei,Lun Chunhua, Yang Zhen.Bullet Velocity Measurement System Based on Photoelectric Signal Conversion Principle and Single Chip Microcomputer[J].Mechanical Management and Development,2008,23(6):29-30.

[5]. Zeng Qingjun,Liu Li,Huang Qiaoliang.Optical Fiber Transceiver with Remote Monitoring Function and Data Transmission Method[J].China:Jiangsu University of Science and Technology invention,, 200910234398,2009,11,24.

[6]. Ding Keqing.Research on The Application of Virtual Simulation and Dynamic Evaluation Method for The OperationState of Large Hoisting Machinery[R].Project supported by "11th Five-Year"(2006BAK02B04)Research Report.CSEI,2009. 